\title{
Activité et contrôle de l'information sur un intranet d'entreprise : censure et régulation du discours sur le stress au travail
}

Activity and Information Control within a Company Intranet: Censorship and the Regulation of the Discussion of Stress at Work

\section{David Douyère}

\section{CpenEdition}

Journals

Édition électronique

URL : http://journals.openedition.org/edc/1037

DOl : 10.4000/edc. 1037

ISSN : 2101-0366

Éditeur

Université Lille-3

\section{Édition imprimée}

Date de publication : 1 décembre 2009

Pagination : 41-56

ISSN : 1270-6841

\section{Référence électronique}

David Douyère, «Activité et contrôle de l'information sur un intranet d'entreprise : censure et régulation du discours sur le stress au travail », Études de communication [En ligne], 33 | 2009, mis en ligne le 01 décembre 2011, consulté le 20 avril 2019. URL : http://journals.openedition.org/edc/1037 ; DOI : 10.4000/edc. 1037

Ce document a été généré automatiquement le 20 avril 2019.

(c) Tous droits réservés 


\title{
Activité et contrôle de l'information sur un intranet d'entreprise : censure et régulation du discours sur le stress au travail
}

\author{
Activity and Information Control within a Company Intranet: Censorship and \\ the Regulation of the Discussion of Stress at Work
}

David Douyère

1 Que devient la connaissance que des acteurs veulent diffuser de leur métier quand la publication sur un dispositif numérique d'information, un intranet, vient mettre en question la «dicibilité » de cette information et sa réception possible, et les amène à transformer celle-ci ? Le dispositif de partage de connaissances destiné à décrire et à orienter l'action n'est plus alors orienté seulement par la transparence qu'il semble annoncer et par la libre expression que l'outil paraît rendre possible (GERMAIN, MALAISON, 2004), mais semble soumis à des enjeux « politiques » (BOMBARDE, 2000) qui conduisent à remanier, à laisser de côté certains aspects, soit que ceux-ci ne correspondent pas à la représentation que souhaite donner d'elle-même l'entreprise, soit qu'ils ne permettraient pas une bonne diffusion ou une bonne réception des contenus proposés. La production de l'information suppose alors une " sélection de l'information » et une compréhension à la fois du cadre d'expression, dans un contexte fortement régulé, notamment sur le plan langagier (BORZEIX, FRAENKEL, 2001) et du public destinataire; l'activité éditoriale qui l'organise s'effectue dans un cadre prescrit, et l'intranet, fut-il collaboratif, n'échappe pas à la règle.

2 Cette relation entre information et communication sur des questions « sensibles » liées à l'activité professionnelle nous semble pouvoir être interrogée ici à travers le cas de la contribution d'un groupe d'acteurs à un intranet professionnel métier, accessible à tous les salariés d'une entreprise, consacré aux questions d'hygiène, santé, conditions de travail (HSCT), autour de la question du stress au travail. Préventeurs (spécialistes de 
l'HSCT conseillers de directeurs d'unités opérationnelles), experts sécurité, chargés de sécurité, managers et médecins du travail délibérant au sein du comité éditorial d'un site intranet (indépendant du comité HSCT de l'entreprise) s'aperçoivent que leur activité de représentation de l'activité professionnelle destinée à orienter cette même activité se trouve comme filtrée par le dispositif qui est censé la permettre. Aux prises avec le système d'information, les acteurs opèrent et voient s'opérer une régulation de la connaissance communiquée, impliquée par la diffusion même de l'information. Des contraintes d'ordre multiple - prise en compte du public, intégration en amont de dimensions juridiques ou managériales, perception du caractère potentiellement « choquant » de certains discours - vont peser sur leur énonciation et les discours dont ils vont alimenter l'intranet.

Nous proposons de considérer deux situations de "filtrage " ou de "censure », pour autant que ce dernier terme puisse être appliqué à l'entreprise - et non seulement à l'espace public -, de l'information, liées à la question du stress au travail, qui montrent ces «positions limites» de la publication sur un intranet. Elles rappellent la double «tension», "politique et organisationnelle» d'un intranet (GUYOT, 2006, 89) et conduisent à reconsidérer l'idée selon laquelle un système d'information pourrait, pleinement, communiquer l'information, hors de toute considération sociale ou politique. Qu'un outil de gestion de contenu (CMS, content management system), outil d' « expression » sur un site intranet puisse ne pas permettre de publier ce que l'on veut, mais que son utilisation soit soumise à des règles de sélection et d'expression de l'information, qu'une entrave au discours puisse ainsi apparaître, voilà qui nous paraît réinsérer ces outils dans le cadre plus large de la régulation des discours et de la communication en organisation (OLIVESI, 2002), montrer comment l'utilisation du système technique est soumise à des règles sociales, comment, enfin, la connaissance n'est pas neutre, mais potentiellement efficiente, et porteuse d'enjeux de pouvoir, ce qui amène à penser l'idée d'une « politique de la connaissance » (DOUYERE, 2005 b) prenant en compte l'incidence sociale de la diffusion des savoirs.

\section{Une « mission » de diffusion de connaissances en santé et sécurité au travail}

4 Créé en 2002 dans le sillage de la dynamique intranet (cf par ex. DAILLIER, 2002), rationalisation donnée comme «libre expression» de la créativité individuelle (BEAUDOUIN, CARDON et al., 2001) favorisant l'innovation ${ }^{1}$, qui, reprenant l'essor et les supposées valeurs du web (FLICHY, 2001), traverse l'entreprise Selenis, entreprise française de stature internationale spécialisée dans les services à base technique, le site intranet Action-Prévention propose aux salariés de l'entreprise, mais avant tout à ses préventeurs et médecins du travail, plusieurs centaines de pages consacrées aux risques liées à l'activité professionnelle, à la façon de les recenser, de les analyser, de les prévenir, et associe les différents métiers de l'entreprise ayant trait à la santé et à la sécurité au travail dans une perspective de transformation de l'activité professionnelle, par l'intermédiaire du travail des préventeurs.

5 Chargé de concevoir et de mettre en place une politique de prévention des risques professionnels auprès du directeur d'unité, les préventeurs de Selenis utilisent ce site comme un guide pour trouver de l'information réglementaire et législative, parfois 
technique, sur des risques que souvent ils connaissent peu, et sur lesquels ils sont interrogés ou auxquels ils se trouvent confrontés dans l'analyse des risques (établissement $\mathrm{du}$ " document unique », notamment) de leur unité. Leur activité consiste dans une veille informationnelle, l'établissement de plans de prévention, la recommandation ou la commande d'équipements de protection individuelle, l'analyse des accidents, la formation et l'information des managers et des salariés, en vue de la prévention des risques dans l'entreprise. À partir de 2000, avec la création de cette fonction, l'entreprise prend en charge la formation, l'information et la «mise en réseaux » d'échange des préventeurs (DOUYERE, 2006).

6 Proposant une « encyclopédie de la santé et de la sécurité au travail » abordant thème par thème les grands risques rencontrés, le site Action-Prévention se présente comme un référentiel en santé et sécurité, mais sans caractère contraignant ni normatif, visant à susciter une réflexion et une "démarche sécurité » davantage qu'à imposer des règles qui, appliquées, ne seraient toutefois peut-être pas comprises. Le site intranet est donc orienté vers l'amélioration (la «montée en compétences ») de l'activité des acteurs de la santé au travail mais aussi des managers, sinon même des salariés de Selenis, qui ont tous accès au site. Cette communication de connaissances, d'analyse et d'outils méthodologiques qui vise à « améliorer la pratique » est réalisée par un collectif d'acteurs rassemblés en un auteur collectif, le " comité éditorial ", dirigé par un ingénieur HSCT (Michel Mongerie), comité dont le débat et l'utilisation d'un outil de gestion de contenu (CMS) fait circuler et met en forme le travail réalisé par chacun seul ou en petit groupe de travail thématique. Une dimension participative anime également le site, sous la forme d'un bouton "contribuer » censé permettre, dès avant le développement du web 2.0 en entreprise, l'apport des intranautes au site.

7 Les contenus mis en ligne sur le site visent donc à cadrer, orienter, permettre, améliorer l'activité professionnelle. Ils répondent à une logique de service informationnel (DURAMPART, 2004) pour la construction du bien commun au travail. En ce sens, ils procèdent d'une dynamique « altruiste » qui renvoie à l'aspiration du métier, qui inclut la préoccupation de la santé des salariés. Le préventeur ou le chargé de sécurité ont quasiment, en effet, une « mission » professionnelle, et cette communication des savoirs envisagée sur les réseaux numériques n'est pas neutre ; elle possède d'une certaine façon une dimension «humaniste». En ce sens, les membres du comité éditorial ont le sentiment non pas de «faire de la communication » mais de diffuser des connaissances utiles à la pratique, en vue de l'amélioration de la santé des salariés. Les contributeurs du site intranet Action-Prévention préfèrent d'ailleurs parler de "message» et de «bonnes pratiques » destinés aux acteurs de la sécurité, que de « communication ». Le dispositif de publication numérique est donc un outil au service de la sécurité, destiné à changer l'activité. La problématique de l'auto-censure ou de l'anticipation des réactions du «public» et des jeux de pouvoir des acteurs prend donc un sens particulier: «le message » en question n'est pas entièrement malléable puisqu'il est soumis à une vérité " objective », "scientifique » ou technique, et réfère à une activité qui le précède et le suit. La régulation de ces discours n'est donc pas sans effets: elle pourra avoir une incidence sur l'action qui peut s'ensuivre, ou non; elle est par ailleurs la résultante des débats sur la stratégie à adopter pour une transformation de l'action en matière de prévention des risques.

8 Nous nous proposons de considérer ici un système éditorial et des situations afférentes observées ${ }^{2}$ pour les interroger et proposer une réflexion sur la sélection et la " censure » 
de l'information en entreprise ainsi que sur la forme conférée aux connaissances présentées, qui peut en affirmer ou en atténuer le sens et la portée. L'enjeu des deux cas que l'on va lire est la réception par les managers de l'entreprise des préconisations en termes de sécurité. Le comité éditorial du site intranet est donc le lieu d'un débat sur ce qui peut être dit ou non, et sur les meilleures façons de dire, ou les façons de dire au mieux, en fonction des contraintes, soit un travail, mené par des non-professionnels de la communication, sur le mode de communication des savoirs proposés. Il atteste d'une tension entre l'activité professionnelle et la sécurité, la première ayant du mal à intégrer la seconde comme une priorité absolue, dans une logique d'augmentation des rythmes de travail et de l'attente en terme de profit et de productivité (GOLLAC, VOLKOFF, 2000), la sécurité étant perçue comme un obstacle à la réalisation du travail dans les meilleurs délais, et un coût supplémentaire. En écrivant sur le site Action-Prévention, et en veillant à ce qu'ils écrivent, les acteurs négocient la place de leur activité et de leur champ dans l'entreprise, dans la durée, dans une perspective de «bien commun »- mais censurent à ce prix une partie de leurs connaissances ou de leurs préconisations.

\section{Lissage informationnel et atténuation : une régulation des discours en entreprise}

9 Si le travail éditorial mené par les vingt membres, environ, du comité éditorial, autour d'un dispositif socio-technique hybride mêlant plateforme de gestion de contenu (CMS), conférences téléphoniques, et rencontres, au fil des réunions thématiques ou bimensuelles de travail, échanges et séances de travail téléphonique, puis solitaires (DOUYERE, 2008), mettant à disposition le contenu produit, concourt à conférer au contenu du site une apparence homogène et claire, il inclut également un certain traitement de l'information, un lissage des scories et saillies, qui participe de la représentation des possibilités des discours sur la sécurité au travail chez Selenis. Une certaine difficulté d'expression, ou une prudence à l'égard de et dans l'expression, manifeste dans l'élaboration éditoriale du site touche d'une part les accidents, cœur noir des métiers de la prévention (DOUYERE, 2005 a), d'autre part les «questions embarrassantes », i.e. les sujets à propos desquels certains préventeurs ou médecins du travail pourraient être tentés d'avoir un point de vue différent de celui de l'entreprise. Abordons un exemple concret de partage de connaissances altéré ou limité par la possibilité d'en tout dire sous la forme de la question du stress, qui montre les limites, les zones d'altération et de nécessaire compromis du discours dans l'institution.

\section{L'incidence politique des connaissances communiquées : le cas du stress dans l'entreprise}

10 Relevant des risques dits " psychosociaux ", le stress fait partie intégrante du domaine de l'HSCT, et concerne tant les médecins de prévention que les préventeurs. Thème de travail du comité national HSCT de Selenis en 2002-2003, qui a constitué une commission spécifique, le stress chez Selenis fait, au premier semestre 2003, l'actualité dans la presse grand public ${ }^{3}$. En 2004 paraît un pamphlet consacré à cette question chez l'éditeur JeanPaul Gawsewitch, dans la collection "Coup de gueule». Le site Action-Prévention, indépendant des instances HSCT de l'entreprise (du comité national HSCT comme de la 
direction des ressources humaines), a inscrit dès sa conception le thème stress à son programme, et l'a mis en ligne, après plusieurs mois de travail, début juin 2003.

Dès le début de sa conception, le thème a fait l'objet de débats vifs au sein du groupe thématique concerné. La phase de validation du thème, toutefois, a également laissé apparaitre de profondes divergences de fond, et une volonté très nette, et affirmée, d'atténuer le propos. Comme le relève un conseiller local HSCT «Dire, oui, il faut parler du stress, tout le monde était d'accord, mais de, d'écrire le dossier, ça c'était une autre paire de manches! » (Bertrand G., conseiller local HSCT, entretien du 23.01.03).

12 Ainsi Annie M., médecin du travail formée au Cnam à la psychodynamique du travail de C. Dejours, et par conséquent assez critique à l'égard des pratiques de l'entreprise, explique-t-elle à propos d'un témoignage sur le stress dans une filiale de Selenis (région Rhône Alpes) publié sur le site Action-Prévention, à partir d'une enquête à laquelle elle a collaboré, qu'il " y a eu une certaine censure, on m'a demandé de reprendre... de reprendre les conclusions de l'enquête " [ton lent et résigné] (Annie M., entretien du 25.02.03) consignée dans le texte écrit par sa collègue de travail. Le texte se terminait, en effet, par les conclusions suivantes :

"Certains points relevés par l'enquête ont été pris en compte, notamment au niveau de l'accueil et de la formation initiale. Il n'en reste pas moins que les redéploiements forcés vers ce type de métier restent mal vécus. De plus l'organisation du travail semble se rigidifier. Un certain degré de lassitude voire d' usure professionnelle commence à apparaitre chez les plus anciens. Elle est aggravée par le peu de perspective professionnelle offerte à ce personnel. »

(Action-Prévention, encyclopédie, thème stress, rubrique témoignage, « Une enquête sur les plateformes e-Selenis», Monique Fraysse, «en conclusion», version intermédiaire non publiée sur le site, site temporaire, du 31.03 .03 .../tmp/stress/p1042115433049.htm ; nous soulignons en italique les points qui ont pu paraître problématiques)

Après discussion en comités, et réécriture, ce texte est devenu :

Certains points relevés par l'enquête ont été pris en compte. Des actions ont été menées au niveau de l'accueil et de la formation initiale. Des améliorations sont encore à apporter sur d'autres points : le vécu du redéploiement, l'organisation du travail, les perspectives professionnelles.

(e-Prévention, encyclopédie, thème stress, rubrique témoignage, "Une enquête sur les plateformes e-Selenis ", Monique Fraysse, "Les suites de l'enquête ", version publiée, consultée le $25.06 .03 \quad-.$. /site/stress/p1042115433049.htm; nous soulignons)

14 On le voit, au cours du travail " éditorial ", toute la partie négative ou critique - qui montrait, en tout cas, une difficulté persistante - de la conclusion a été gommée, sans que l'information factuelle soit totalement effacée, au sens strict; toutefois, le mot de la fin est désormais positif, et non plus inquiétant, et la dimension possiblement vindicative a disparu : la perspective a changé. C'est un exemple du « lissage », fort, ici, et du caractère positif conféré, autant que possible, au texte, effectués par le comité éditorial ActionPrévention.

15 La position forte de certains médecins du travail, remise en cause ici, porte sans doute la trace d'un certain conflit « historique » entre la médecine du travail et l'entreprise, et traduit l'engagement de certains médecins, "militants» (PIOTET, 2002, 215) pour l'amélioration des conditions de travail des salariés, qui conduira certains d'entre eux à démissionner de leurs fonctions, en 2009. 


\section{Anticiper la réception et pondérer l'impact sur l'activité : la censure}

16 L'édition du texte "La prévention du stress au travail : la prévention primaire » au sein du thème «Stress » du site intranet Action-Prévention suscite un vif débat en 2002-2003, puis un remaniement notable du texte, après un travail effectué sur une durée de six mois. En effet, la version initiale, préparée, comme à l'ordinaire, par un groupe de travail thématique au sein du comité éditorial, comportait (version de travail du 22.01.03) les mentions suivantes qui, pour celles en italiques, seront supprimées (version publiée du 25.06.03):

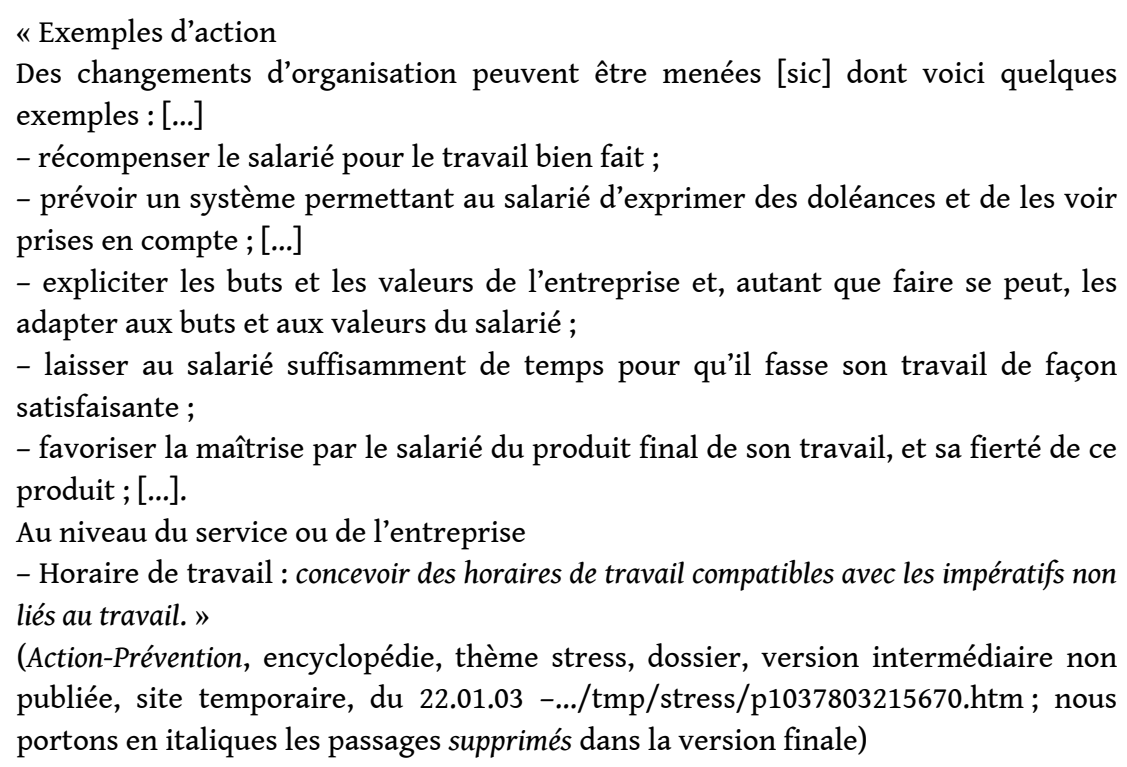

En «contrepartie » de ces suppressions, la version finale proposera les ajouts suivants, soulignés ici :

"Il n'y a pas de démarche universelle et les paragraphes suivants ne font que donner des exemples d'amélioration.

Exemples d'action :

- Favoriser la contribution du salarié dans l'organisation de son activité. Tenir compte de ses remarques. [...]

- Donner au salarié des signes de reconnaissance pour le travail bien fait. Au niveau du service ou de l'entreprise: [...]

- Amélioration des horaires de travail : lorsqu'elles existent, utiliser les marges de manoeuvre par rapport aux exigences temporelles de l'activité. "

(Action-Prévention, id., version publiée, consultée en ligne le 25.06 .03 -.../site/stress/ p1037803215670.htm; les passages ajoutés par rapport à la première version sont ici soulignés).

La réécriture a gommé ce qui dans le texte initial mettait le plus en cause les pratiques ordinaires de l'entreprise et paraissait "demander l'impossible ». Certains éléments reviennent toutefois, donnés en un sens plus général et collectif (« donner aux salariés des signes... »). L'utopie sociale inacceptable du texte, aux yeux du comité éditorial, en ce qu'il opèrerait un quasi-renversement des pratiques managériales, est repoussée afin que ce texte ne vienne pas à constituer une référence pour d'autres discours, qu'il viendrait alors rendre légitimes. La censure évite ici que ne soient produits des signes qui 
pourraient recéler des (ou être investis de) significations orientant l'activité dans un sens différent que celui vers lequel le management de l'entreprise la conduit.

« Ah oui, mais, moi, dès que j'avais vu ce texte, j'avais appelé Michel [le responsable du comité éditorial] en lui disant «Attend, euh, on peut pas publier un truc pareil, parce que, nous, dans les unités..., Comment on va... ? Comment on va gérer ça, quoi ? Parce que... enfin. Si on estime que les gens vont voir ce... Ils vont nous dire... "Bon, bah, alors, attendez..." Et, bon. Bah, ça a été repris [i.e. retravaillé]. Je trouve que, maintenant, ça y est. Ça commence à... [...] C'était surtout pas réaliste par rapport à ce qu'on peut connaître sur le terrain.» (Denise E., responsable RH d'unité, entretien du 17.04.03; nous soulignons, italiques)

19 La pensée de l'usage qui peut être fait d'un texte, de la façon dont on peut s'en recommander, de son "invocabilité", conduit ainsi à le retoucher. Le responsable du comité éditorial du site intranet Action-Prévention explique les motifs de la retouche du texte en comité :

"C'est pas réceptif [i.e. recevable] par un manager aujourd'hui [...]. Il y a un certain nombre de choses qui sont, à mon avis, pas acceptables par un manager. Quand on dit " concevoir des horaires de travail, euh... en tenant compte des contraintes extraprofessionnelles des gens... » On peut pas l'écrire comme ça! [...] Si on marque ça sur le site, je veux dire, quelque part, on se décrédibilise vis-à-vis de la partie, euh, managériale. Qui est aussi une de nos cibles, quand même. Donc y faut euh... Enfin une de nos cibles... Et puis je pense pas qu'on puisse tenir très longtemps à tenir ce type de discours sans... dans une entreprise, on est quand m... on est aussi dans une entreprise... » (Michel M., responsable du comité éditorial du site, entretien du 27.01.03, nous soulignons, italiques)

La double contrainte de la politique éditoriale et de la logique d'entreprise, et le souci de la construction de la professionnalité du site (devenir une référence) comme de la fonction de prévention, amènent donc à contrôler les discours. Ce qui gêne notamment dans ce texte, c'est, bien entendu, la place accordée au salarié :

«La présentation qui est faite est... tellement... orientée côté salarié [...] qu'elle en devient inaudible pour le... manager, [...] Alors, inaudible, avec, [...], le risque que ça décrédibilise complètement le dossier, alors qu'il y a des choses intéressantes et importantes à faire, et deuxièmement, euh, que quelque part ça nous soit reproché un jour comme étant, euh..., un discours, euh, quasiment syndical, quoi... [...] Dire les choses comme ça, c'est quand même, euh, enfin, risqué [ou : risquer], [...] on va heurter les gens et du coup y vont pas... y vont pas adhérer au reste, quoi... C'est ça qui est un peu gênant. » (Michel M., idem)

21 Assujettis à une cible et à un contexte, les propos doivent être remaniés pour être «admissibles », pour les managers, mais aussi pour les salariés (et les médecins) engagés dans une perspective davantage "sociale». La censure exercée ici est un contrôle éditorial destiné à optimiser la diffusion de la publication: conserver une crédibilité oblige à limer ses discours, à les conformer au langage recevable, à s'approcher de ce qui peut être entendu, et que l'on suppose. L'ajustement, le rejet de certains propos se fait $a$ priori - mais en connaissance des discours qui circulent dans l'entreprise, et d'une partie des éventuels destinataires. "Tout ne peut pas être dit" et la mise en place d'un processus technique (cms, groupware, courriers électroniques, réunions téléphoniques...), l'appui sur une communauté de métier et de préoccupation, ne peuvent autoriser de diffuser par écrit un discours que l'organisation ne peut recevoir. Le caractère hybride du dispositif communicationnel utilisé (parole et écriture) permet peut-être précisément ici une régulation atténuée des contenus diffusés que la publication directe sur le CMS ne permettrait pas. 
22 La censure, par le collectif, dans le débat et le "dialogue », du texte est effectuée au nom même du principe de communication des savoirs en sécurité adopté sur le site. Le regard sur l'activité et son degré de modification possible amène ici à remanier le texte, afin d'éviter qu'il ne «fasse signe». Le site ne prend pas le risque de la contradiction qui lui serait opposée, mais y répond d'avance. Politique « réaliste» de publication ou censure d'une approche "scientifique", mais politiquement marquée, du stress au travail? La communication des savoirs élaborés en collectif ne se passe pas, on le voit, sans filtrage ni anticipation de sa réception auprès des salariés, du fait notamment de la logique de construction de la notoriété et du caractère de référence du site intranet, qui ne doit pas se trouver rejeté par les managers. Une logique de média, incluant une prise en compte du public, et de l'image du site dans la durée, est ici à l'œuvre. Le système d'information tourné vers l'activité n'est pas un medium sans altération, mais inclut un filtrage éditorial, sinon une censure, et (pré)inscrit l'activité dans la conception même du contenu qui vise à l'accompagner.

\section{Conclusion}

«Sur le site Action-Prévention, quand même, faut toujours donner un aspect positif, quoi, il faut... [sourire moqueur dans un souffle], oui... Alors que bon... Y a bien quand même des choses qui se passent mal à Selenis en ce moment... [ton plus ferme, plus sérieux sur la fin de la phrase]. C'est vrai que c'est un peu, euh, comment dire,... on atténue (sourire dans un souffle). On a tendance sur ActionPrévention, à... à atténuer [mot dit plus lentement], euh, en particulier les... les effets sur la santé. » (Annie M., entretien du 25.02.03, elle souligne et nous soulignons en italiques)

Annie M. voit ainsi dans le travail éditorial du comité quelque chose qui oriente les textes et atténue leur gravité éventuelle, ce à quoi elle confère un sens politique. Le travail et le choix des mots, la reprise des phrases, participent en effet de ce lissage sémantique qui vise à éroder le propos, et à le rendre "acceptable", non choquant. En effet, le site intranet représente l'institution, et s'adresse à elle - les préventeurs sont les conseillers des managers. La pondération utilisée ici, l'art de la modération, relèvent d'une écriture d'entreprise, de l'euphémisation, dans un contexte de régulation et de contrôle des discours.

Le comité éditorial du site intranet Action-Prévention constituerait ainsi, autant qu'une cellule de production ou de collation de connaissances (DOUYERE, 2008), un espace projeté de régulation des discours et de l'expression écrite de la pensée, afin que ne soit dit (écrit) que ce qui peut l'être. L'échange au sein du comité est en effet un sas de filtrage : un sujet ne paraîtra pas s'il n'est rendu "recevable " (« réceptif », comme le dit un membre du comité éditorial) par les managers, la ligne hiérarchique et les directeurs des ressources humaines. Les membres du comité éditorial du site Action-Prévention sont tout à fait conscients de ce fait.

Faut-il parler de "censure ", alors que l'on se trouve dans l'espace privé de l'entreprise, dont le contrôle est en partie légitime? Ou de simple «travail éditorial »? Voire, comme le fait, avec un sourire ironique, le responsable adjoint du comité éditorial du site intranet, médecin du travail, de "sélection de l'information »? Si la censure est ce qui «tend à priver d'existence publique tout ou partie d'un texte, d'un discours ou d'un message quelconque » (DURAND, 2006, 12), c'est bien de censure dont il faut parler, quelle que soit la mesure de cet espace public (dans l'entreprise même, au sens de «l'espace 
public partiel ou incomplet» dans les grandes entreprises avancé par B. Floris (1995, 129)), censure qui s'intrique toutefois à la fois à l'élaboration et à la délibération «scientifique » (ie. sur le fond) du comité éditorial, à l'issue desquelles des éléments de connaissance se trouvent partiellement écartés, et au travail éditorial qu'il mène sur les textes (constitution de la lisibilité, faciliter l'appropriation par le public). Cette censure porte sur l'écrit, non sur la parole dans l'entreprise. Comme le dit le responsable du site intranet : "Oui, censure, bah, $y$ a des choses qu'on... Oui, oui oui [...] Oui, oui y a des trucs qu'on dirait peut-être, mais qu'on met pas... [sur le site intranet] » (Michel M., entretien du 27.01.03, nous soulignons).

Des régimes de vérité (scientifique et médical, managérial dans l'entreprise) et de dicibilité, au sens de représentation de ce qui peut être dit, et des modalités d'expression ( lissée » ou explicite), sinon même des langages, différents, sont ici en jeu, et en conflit, qui ne doivent pas voiler la dimension de censure (un ne-pas-dire... sous cette forme), qui résulte précisément de la tension entre ces éléments. Cette dimension censoriale, naturellement, ne concerne pas uniquement l'entreprise : toute situation sociale humaine est marquée par cette "limitation du dire " contextuelle, inscrite probablement dès et dans le langage, et d'aucuns ont même pu en faire une propriété de l'appareil psychique.

Le choix de l'information, "et tout choix est à la fois élection [...] et exclusion [...]» (DURAND, 2006, 17), la réécriture et le « lissage » des textes conduisent ici à une censure, ou comme l'écrit le poète Bernard Noël $(1990,180)$, à la suite peut-être du psychanalyste Jacques Lacan, à une "sensure", entendue comme ce qui "vide l'expression pour la rendre inoffensive, démarche beaucoup plus radicale et moins visible ", attestant du lien, parfois oublié, entre l'information et le sens (FLORIS, 2004, 86), et le politique. L'information pour l'activité n'est donc pas sans enjeux axiologiques et sémantiques, et s'avère aussi contrôlée que l'activité elle-même, quelque soit la liberté apparente conférée aux dispositifs communicationnels utilisés.

\section{BIBLIOGRAPHIE}

Beaudouin, V., Cardon, D., Mallard, A. (2001), « De clic en clic, créativité et rationalisation dans les usages des intranets d'entreprise », Sociologie du travail, 43/3, p. 309-326.

Bombarde, S. (2000), Intranet, pour une communication réussie, Paris : Stratégies.

Borzeix, A., Fraenkel, B. (dir.), (2001), Langage et Travail, Communication, cognition, action, Paris : Cnrs.

Daillier, A. (2002), « Utilisation de l'intranet à France Télécom », réseau cndp, Réseaux humains / réseaux technologiques, des intranets de formation, Poitiers : CRDP Poitou Charente, p. 85-90 et 90-94.

Douyère, D. (2008), « Publication collaborative sur un intranet et processus éditorial », E. Broudoux, G. Chartron (dir.), Traitements et pratiques documentaires, vers un changement de paradigme ?, Document numérique et société, $2^{\mathrm{e}}$ conf., Paris : ADBS, p. 223-240. 
Douyère, D. (2006), « Collectifs et diffusion des connaissances au travail : le cas de la fonction hygiène et sécurité chez Selenis », Terminal, n97-98, p. 133-144.

Douyère, D. (2005 a), « Tic et partage de connaissances en entreprise : une impossible injonction à l'expression? L'expression des connaissances au travail dans le domaine des métiers de la sécurité des conditions de travail - une étude de cas ", gdr « tic \& Société », cnrs, $3^{e}$ Doctoriales, 2005,

http://gdrtics.u-paris10.fr/pdf/doctorants/papiers_2005/David_Douyere.pdf

Douyère, D. (2005 b), Communication et politique de la connaissance, thèse de doctorat en sciences de l'information et de la communication, sous la dir. de Roger Bautier, Université Paris 13.

Durampart, M. (2004), « Les services informationnels, SIO et organisation du travail », Sciences de la société, $\mathrm{n}^{\circ} 63$, p. 151-164.

Durand, P. (2006), La Censure invisible, Arles : Actes Sud.

Flichy, P. (2001), L'Imaginaire d'Internet, Paris : La Découverte.

Floris, B. (2004), « L'information a-t-elle du sens ? La question du sens dans les Sic », Sciences de la société, $n^{\circ} 63$, p. 75-92.

Floris, B. (1995), « L'entreprise sous l'angle de l'espace public », I. Pailliart (dir.), L'Espace public et l'emprise de la communication, Grenoble : Ellug, p. 119-138.

Germain, M., Malaison, C. (2004), L'Intranet dans tous ses états, Québec : Isabelle Quentin éditeur.

Gollac, M., Volkoff, S. (2000), Les Conditions de travail, Paris : La Découverte.

Guyot, B. (2006), Dynamiques informationnelles dans les organisations, Paris, Lavoisier.

Le Moënne, C. (1995), « Espace public et entreprises : penser la sphère professionnelle »,

I. Pailliart (dir.), L'Espace public et l'emprise de la communication, Grenoble : Ellug, p. 138-162.

Noël, B. (1990), « La pornographie », Le Château de Cène, Paris : Gallimard.

Olivesi, S. (2002), La Communication au travail, Grenoble : PUG.

Piotet, F. (2002), « Médecins du travail », La Révolution des métiers, Paris : PUF, p. 291-316.

\section{NOTES}

1. Déclaration du président de Selenis dans plusieurs médias internes et externes, en 2000.

2. Au cours d'une recherche conduite sur trois ans, mêlant entretiens, observation nonparticipante de situations de travail (réunions téléphoniques ou en présence, formations, contribution à l'intranet, notamment), analyse de documents et d'archives.

3. Il fait de nouveau l'actualité en 2009 suite à plusieurs suicides survenus dans l'entreprise. 


\section{RÉSUMÉS}

Alors même qu'un dispositif censé permettre une communication des connaissances en matière de santé au travail est mis en place sur un intranet d'entreprise afin de soutenir l'activité des responsables de la mise en place de la politique de sécurité au travail (préventeurs), l'analyse des différentes versions des textes proposés par le comité éditorial du site intranet Action-Prévention ainsi que des entretiens avec ses membres laissent apparaître une atténuation des propos, un lissage des textes et une censure de certains contenus concernant le stress au travail, au motif qu'ils pourraient heurter les managers de l'entreprise. Une régulation des discours apparait donc, qui contraste avec la libre diffusion des savoirs proposée en apparence par l'outil de gestion de contenus alimentant l'intranet. L'activité d'information rencontre ici une dimension politique, comme celle du sens et de la portée de ces informations pour l'activité.

A system supposedly permitting a dissemination of information on health in the workplace is created on a company intranet in support of those responsible for workplace safety policies. However, the analysis of different versions of the texts presented by the editorial committee on the intranet site "Action and Prevention", as well as interviews with its members, reveals a mitigation of these remarks, a glossing over of the texts and censorship of content concerning stress in the workplace, the reason being that it could do damage to the company managers. A form of censorship is therefore brought to light that is in direct contrast with the free distribution of knowledge that appears to be proposed by the intranet site. The sharing of information becomes political here, as do the meaning and scope of this information within the company.

\section{INDEX}

Mots-clés : censure, intranet, connaissances, santé au travail, information, discours, entreprise, site

Keywords : censorship, intranet, knowledge, health at work, information, speech, business, area

\section{AUTEUR}

\section{DAVID DOUYÈRE}

David Douyère est maître de conférences en Sciences de l'Information et de la Communication à l'Université Paris 13 (Villetaneuse), chercheur à la MSH Paris Nord et membre du Labsic. Ses recherches portent sur la communication des connaissances par les réseaux socio-techniques et les supports écrits, numériques ou imprimés, en entreprise (dans le domaine hygiène, sécurité, conditions de travail) et dans la société (encyclopédies, édition politique, édition numérique) en liaison avec la notion problématique de « société de la connaissance ». 